\title{
Microstructural Characterization and Image Analysis in Ex-Service Ethylene Pyrolysis Tubes
}

\author{
A.C. McLeod ${ }^{1}$, C.M. Bishop ${ }^{1}$, K.J Stevens ${ }^{2}$ and M.V. Kral ${ }^{1}$ \\ ${ }^{1 .}$ University of Canterbury, Christchurch, New Zealand. \\ 2. Quest Integrity NZL Ltd., Wellington, New Zealand.
}

Ethylene is used extensively in the production of plastics, cabling, and automotive products. It is typically produced by the thermal cracking (pyrolysis) of more complex hydrocarbons, such as naphtha or ethane, at 950 to $1100^{\circ} \mathrm{C}$ inside $\mathrm{HP}$ alloy tubes $(25 \% \mathrm{Cr}-35 \% \mathrm{Ni})$ within an ethylene pyrolysis furnace. The main contributor to the failure of ethylene pyrolysis tubes is carburization of the tubes, which causes an increase in internal volume, a loss in weldability and a reduced ability to withstand thermal cycles [1-4]. Knowing the level of carburization of a tube in-situ can assist in remaining life estimates based on Finite Element Modeling, thermography, and fracture mechanics. Due to the changes in the microstructure and magnetic properties of the tubes over their service life, the level of carburization can be detected non-destructively using eddy current probes [3] and a tube crawler system [5]. However, the eddy current system requires calibration on ex-service tubes that have had their microstructure, mechanical properties and magnetic response characterized.

The growth of chromium carbides and other chromium-rich phases due to carbon ingress results in a depletion of chromium in the matrix. In the as-cast condition the matrix is paramagnetic, but as carburization progresses it tends towards the binary Fe-Ni system and becomes progressively more ferromagnetic [3, 4, 6-8]. The change in bulk permeability of the tube is measured by the eddy current system. As such, the volume fractions of the phases that contribute to the matrix chromium depletion and the distribution of these phases across the tube wall are of interest. The volume fractions and distributions are being characterized by way of imaging the tube microstructure along radial wall profiles, and using a combination of two programs, ilastik and FIJI, for the segmentation and processing of the images. ilastik is a free software package that enables automated classification and segmentation of batches of images [9].

Initially, BSE (backscatter electron) images were proposed for image analysis, due to their high resolution, wide range of grayscale values for differentiating phases, and relatively short collection time. However, one major feature of BSE images of ex-service tubes proved to be detrimental to the success of automated segmentation using ilastik. A "watermark" effect is often visible in the austenitic matrix of highly carburized tubes when imaging in BSE mode, causing significant gradients in grayscale. Another phase often present in the microstructure of ex-service tubes, the $\eta$-carbide, also exhibits gradients in grayscale across the phase regions. As a result, automated segmentation of the BSE images of highly carburized tubes in ilastik proved to be impossible, as ilastik is unable to distinguish the $\eta$-carbide from regions of the matrix that exhibit this "watermark" effect. Despite testing numerous variations in the mechanical polishing parameters, the "watermark" effect was unable to be minimized. It is possible that it is a feature of the microstructure, for example a strain effect due to the internal stresses created during carburization, as opposed to a polishing artifact that can be removed.

In the present work, EDS (energy dispersive spectroscopy) maps with SEI image overlays are being utilized as the input for image analysis as opposed to BSE images. Using EDS maps ensures that 
polishing artifacts and/or strain variations in the matrix do not cause issues with segmentation in ilastik, while the SEI image overlay allows clear demarcation of the edges of the phase regions and the necessary contrast between phases to be achieved. In particular, as the $\eta$-carbide (chromium-nickelniobium silicide) has a different composition to the austenitic matrix (iron-chromium-nickel), the two phases have two clearly distinguishable colors in EDS maps, allowing ilastik to segment these phase regions with high accuracy, as shown in Figure 1. Another significant benefit of EDS maps is the ability to analyze the chromium content of the matrix. As the matrix chromium content is the link between the microstructure of the tube and its magnetic response, the data collected via EDS mapping will be instrumental in comparing the microstructure with the eddy current NDT response of the tubes [10].

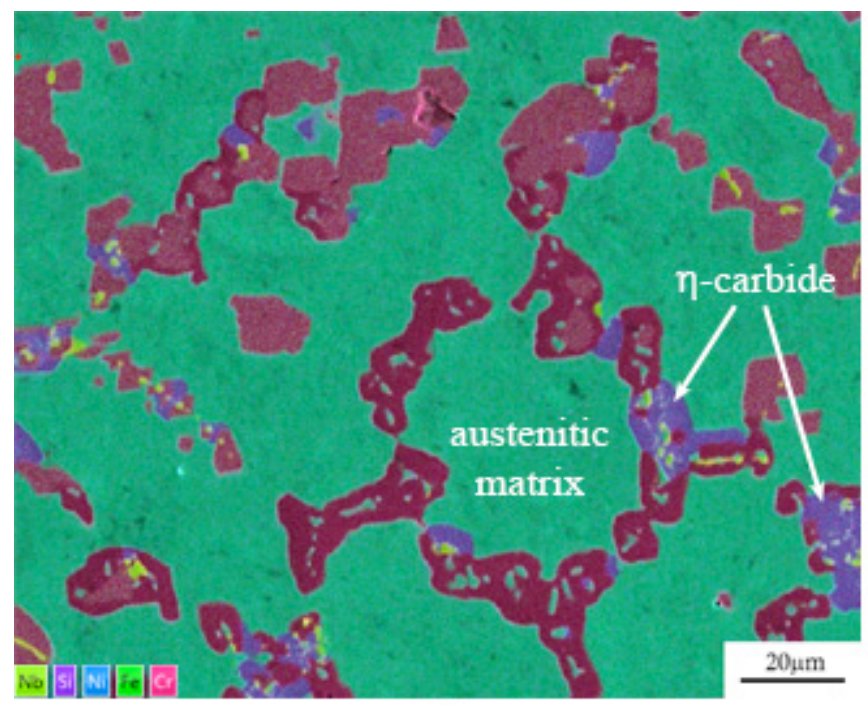

(a)

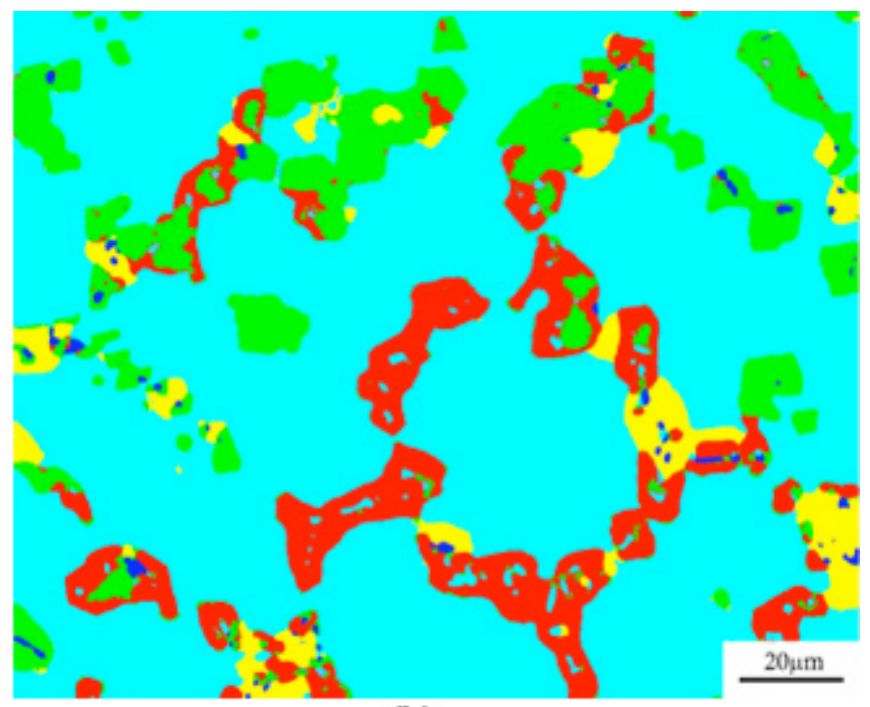

(b)

Figure 1: (a) EDS map of mid-wall region of a highly carburized ex-service HP alloy ethylene pyrolysis tubes, (b) ilastik segmentation of map shown in (a).

[1] D. Jakobi, R. Gommans, Materials and Corrosion, 54 (2003) 881-886.

[2] E. Lang, J. Norton, Commission of the European Communities, Physical Science, PETTEN, EUR $10566 \mathrm{EN}, 1986$.

[3] K.J. Stevens, W.J. Trompetter, Journal of Physics D (Applied Physics), 37 (2004) 501-509.

[4] K.J. Stevens, et al., Journal of Physics D (Applied Physics), 34 (2001) 814-822.

[5] Quest Integrity Group. Ethylene Pyrolysis Tube Inspection System. Available from: http://www.questintegrity.com/technology/Ethylene-Pyrolysis-Tube-Inspection-System.

[6] K.J. Stevens, et al., Current Applied Physics, 4 (2004) 304-307.

[7] K.J. Stevens, et al., Journal of Physics D (Applied Physics), 36 (2003) 164-168.

[8] I.C. da Silva, et al., NDT \& E International, 39 (2006) 569-577.

[9] C. Sommer, et al., 2011 8th IEEE International Symposium on Biomedical Imaging: From Nano to Macro, ISBI'11, March 30, 2011 - April 2, 2011, IEEE Computer Society, Chicago, IL, United states, 2011, pp. 230-233.

[10] This work has been supported by Quest Integrity NZL Limited, and the Ministry of Science and Innovation of New Zealand under contract QINZ1001. Thanks to Andy Saunders-Tack and Charles Thomas of Quest Integrity NZL Ltd for their continued support. 